Pattern Recognition, Vol. 28, No. 12, pp. 1855-1870, 1995

\title{
Polyhedral Object Recognition by Indexing ${ }^{1}$
}

\author{
Radu Horaud and Humberto Sossa \\ LIFIA-IMAG \\ 46, avenue Félix Viallet \\ 38031 Grenoble FRANCE \\ Radu.Horaud@inrialpes.fr
}

\begin{abstract}
In computer vision, the indexing problem is the problem of recognizing a few objects in a large database of objects while avoiding the help of the classical image-feature-to-object-feature matching paradigm. In this paper we address the problem of recognizing 3-D polyhedral objects from 2-D images by indexing. Both the objects to be recognized and the images are represented by weighted graphs. The indexing problem is therefore the problem of determining whether a graph extracted from the image is present or absent in a database of model graphs. We introduce a novel method for performing this graph indexing process which is based both on polynomial characterization of binary and weighted graphs and on hashing. We describe in detail this polynomial characterization and then we show how it can be used in the context of polyhedral object recognition. Next we describe a practical recognition-by-indexing system that includes the organization of the database, the representation of polyhedral objects in terms of 2-D characteristic views, the representation of this views in terms of weighted graphs, and the associated image processing. Finally, some experimental results allow the evaluation of the system performance.
\end{abstract}

Keywords - Object recognition, polyhedral object representation, polynomial graph characaterization, indexing, hashing, feature extraction.

\section{Introduction}

The problem of object recognition in computer vision is the problem of matching object features with image features. Nevertheless, since an object has many features associated with it and since an image contains features that do not necessarily belong to that object, the matching process is a complex one because of the large size of the set of image-feature-to-object-feature assignments. Therefore, in the past, the rationale has been to use constraints - such as the rigidity constraint - in order to maintain the number of assignments as reduced as possible: Therefore, any search process, including exhaustive search, is likely to be fast enough because it has to visit a few thousands of nodes rather than millions.

Various implementations of matching-based recognition of rigid objects take the form of either a search-graph or a search-tree. Examples of search graphs are maximal-clique finding algorithms introduced in computer vision by Ambler \& al. [1], popularized by Ballard \& Brown [2], and applied

\footnotetext{
${ }^{1}$ This work has been supported by the Esprit programme through the SECOND project (Esprit-BRA No. 6769) and by the ORASIS project (PRC Communication homme/machine).
} 
to 2-D object recognition by Bolles \& Cain [3]. Subsequently, the advantage of using trees rather than graphs was stressed by a large number of authors such as Bolles \& Horaud [4], Faugeras \& Hebert [5], Ayache \& Faugeras [6], Grimson \& Lozano-Perez [7], Goad [8], Flynn \& Jain [9], and many others.

However, most of the object recognition methods just mentioned restrict the recognition to object whose exact geometry is known in advance. A more general approach consists of representing both the image and the object by graphs and of casting the recognition problem into the graph matching problem. Graphs are a convenient way of representing features and relationships between these features. Various graph representations have been used by Kim \& Kak [10], Flynn \& Jain [11], Dickinson \& al. [12], and Bergevin \& Levine [13]. However, graph matching is a difficult problem in itself. Whenever the two graphs to be matched have the same number of nodes, graph matching is equivalent to searching for graph isomorphism and polynomial time solutions exist in this case, [14], [15], [16]. It is however rarely the case that the image graph have the same size as the object graph: The problem is therefore equivalent to maximum subgraph matching - find the largest isomorphic subgraphs of the two graphs. So far, solutions proposed for solving the maximum subgraph matching problem involve some form of combinatorial optimization [17], [18].

If many objects rather than a single one (as it has often been the case) are present in a database of objects to be recognized, then the matching-based recognition becomes intractable because the complexity grows substantially with the number of features. An indexing process is crucial whenever the recognition process has to select among many possible objects. Recognition by indexing is therefore the process that attempts to rapidly extract from a large list of objects, those few objects that fit a group of image features while avoiding to establish image-feature-to-object-feature assignments.

Nevatia \& Binford [19] were among the first to describe indexing as a part of an object recognition system. Ettinger [20] described a hierarchically organized object library well suited for indexing. Each object is decomposed into a list of sub-parts. The rationale is that many objects share a common set of sub-parts and what distinguishes one object from another is sub-part relationships - the overall list of sub-parts grows sub-linearly with the number of objects in the library. This idea is applied to flat objects that are described by their outlines.

The idea of using hashing in conjunction with object recognition was introduced by Kalvin \& al. [21]. Outlines of flat objects are described in terms of footprints. The best way to think of a footprint is of an intrinsic curve such as curvature as a function of curvilinear abscissa. The footprint of an object is further decomposed into intervals. Each such interval is described by a set of numbers (the sine and cosine Fourier coefficients, for example) and these numbers are hashed in hash-tables. The indexing itself takes the form of a vote: Each footprint interval detected in the image votes for those objects in the database containing this footprint interval. Finally the object that received the highest vote score is the recognized object. A variation of this method using local frames and geometric hashing was proposed by Lamdan \& Wolfson [22] for solving the matching problem, not the indexing problem.

Following the same idea of hashing, Stein \& Medioni [23] were able to recognize 3-D objects from 3-D data using super-segments and surface-patches as features. Their structural hashing technique retrieves object hypotheses from the database using hash-table indexing. A similar approach was proposed by Breuel [24].

The approach advocated in this paper capitalizes onto the representation of 3-D objects in terms of $2-\mathrm{D}$ characteristic views and of characterizing such a view with a number of weighted graphs. An 
identical graph representation is extracted from the image of an unknown 3-D scene. A polynomial characterization of graphs allows us to organize the "model graphs" into hash tables. Therefore, recognition consists of computing similar polynomial characterizations for the "image graphs" and of indexing in the pre-stored hash tables. Finally, a voting process ranks a number of candidate characteristic views as potential recognized objects.

\subsection{Paper organization}

The remainder of this paper is organized as follows. Section 2 introduces the polynomial characterization of a binary graph that will be used, namely the second immanantal polynomial of the Laplacian matrix of a graph. Then we briefly describe an extension of this representation to weighted graphs. Section 3 describes the graph indexing method that uses this polynomial characterization of graphs. It describes as well an object representation in terms of weighted graphs, the organization of the database of objects to be recognized, and the indexing method itself which is based on hashing. Section 4 describes a representation of 3-D polyhedral objects in terms of 2-D views and a representation of these views in terms of weighted graphs. Section 5 describes how to extract these weighted graphs from images and how to remove irrelevant image data. Section 6 describes a recognition experiment carried out with a set of 9 images of the same scene in the presence of a database of 6 objects. Finally, section 7 draws some conclusions and gives some directions for future work.

\section{Polynomial characterization of a graph}

The method that we propose in this paper in order to achieve indexing uses graphs to represent both images and objects. Let us suppose that one is able to extract a number of graphs from an image and let $G_{1}$ be such an image graph that has the same number of nodes as a graph $G_{2}$ extracted from an object. Such a graph (an image or an object graph) is defined by a set of vertices $V$ and a set of edges $E$. The two graphs $G_{1}=\left(V_{1}, E_{1}\right)$ and $G_{2}=\left(V_{2}, E_{2}\right)$ are said to be isomorphic if there is a bijection $\phi: V_{1} \longrightarrow V_{2}$ such that:

$$
\left(v_{1}, v_{2}\right) \in E_{1} \text { if and only if }\left(\phi\left(v_{1}\right), \phi\left(v_{2}\right)\right) \in E_{2}
$$

If $A_{1}$ and $A_{2}$ are the adjacency matrices of the two graphs, one can easily see that $G_{1}$ is isomorphic to $G_{2}$ if and only if there exists a permutation matrix $P$ satisfying:

$$
A_{2}=P A_{1} P^{-1}
$$

Hence, there are two ways to decide whether two graphs are isomorphic:

1. Find the permutation matrix $P$ that satisfies the equation above.

2. Find an algebraic characterization of the adjacency matrix of a graph that is invariant under a similarity transformation of the adjacency matrix. Such a characterization has been proved to be useful for graph classification. 
One obvious characterization that is invariant under similarity is the characteristic polynomial associated with the adjacency matrix [25], [26]. Indeed we have:

$$
\begin{aligned}
\operatorname{det}\left(x I-P A P^{-1}\right) & =\operatorname{det}\left(P x I P^{-1}-P A P^{-1}\right) \\
& =\operatorname{det}\left(P(x I-A) P^{-1}\right) \\
& =\operatorname{det}(x I-A)
\end{aligned}
$$

Therefore, the similarity of adjacency matrices is a necessary condition for isomorphism. Unfortunately it is far from being a sufficient condition. However, an important idea stems out from this example of graph characterization - one may seek to characterize a graph, up to an isomorphism, by the coefficients of a polynomial associated with that graph. More formally, we seek a polynomial associated with a graph, say $p(G)$ such that:

$$
\left\{\begin{array}{l}
\text { if } G_{1}=G_{2} \text { then } p\left(G_{1}\right)=p\left(G_{2}\right) \\
\text { and } \\
\text { if } p\left(G_{1}\right)=p\left(G_{2}\right) \text { then } G_{1}=G_{2}
\end{array}\right.
$$

Two graphs are said to be equal if they have the same number of nodes and if they are isomorphic. Two polynomials are equal if they have the same degree and if their coefficients are equal. If a polynomial satisfying the above condition exists, it follows that the problem of comparing two graphs of the same size is equivalent to the problem of comparing the coefficients of their associated polynomials. Notice however that graph characterization with a polynomial allows one to state whether two graphs are isomorphic or not but it doesn't provide the node-to-node isomorphic mapping between the graphs. Graph characterization is therefore exactly what one needs for model indexing, i.e., rapidly state whether some sensed data equal some object data. The search of an isomorphic node-to-node mapping is the task of matching and not the task of indexing.

Polynomials that characterize a graph unambiguously up to an isomorphism have been thoroughly studied in the linear algebra literature [26], [27]. Among these polynomials, the second immanantal polynomial - or the $d_{2}$-polynomial - is a good candidate [28].

One may associate the second immanantal polynomial with the adjacency matrix of a graph. There are however some reasons to prefer the Laplacian matrix (defined below) to the computationally simpler adjacency matrix. The Laplacian matrix is positive semidefinite symmetric of rank $n-1$ (if $G$ is a connected graph). Generally speaking, second immanantal polynomials match up well with positive semidefinite matrices. The greater complexity of Laplacian matrices, when compared with adjacent matrices, suggests there may be fewer algebraic accidents [28]. If the time to compute the determinant of a $n \times n$ matrix is $n^{3}$, the time to compute the coefficients of the second immanantal polynomial is $n^{4}$.

The elements of the Laplacian matrix of a binary graph, $L(G)$, are defined as follows:

$$
l_{i j}= \begin{cases}d_{i} & \text { if } i=j \\ -1 & \text { if there is an edge between nodes } i \text { and } j \\ 0 & \text { otherwise }\end{cases}
$$

where $d_{i}$ is the number of graph edges meeting at node $i$ and is called the degree of the node $i$. The interest reader may find in [29] a complete description of the properties of the Laplacian matrix of a binary graph. 
The second immanantal polynomial associated with a $n \times n$ Laplacian matrix of a graph, $L(G)$, can be written in generic form as:

$$
d_{2}(x I-L(G))=c_{0}(L(G)) x^{n}-c_{1}(L(G)) x^{n-1}+\ldots+(-1)^{n} c_{n}(L(G))
$$

The coefficients $c_{o}, \ldots, c_{n}$ of this polynomial are integers and they can be computed using the following formulae which are detailed by Merris [28] ( $n$ is the number of nodes of the graph and $m$ is the number of edges of the graph):

$$
\left\{\begin{aligned}
c_{0}(L(G)) & =n-1 \\
c_{1}(L(G)) & =2 m(n-1) \\
& \vdots \\
c_{k}(L(G)) & =\sum_{X \in Q_{k, n}}\left(\sum_{i=1}^{n} l_{i i} \operatorname{det}(L(G)\{X\}(i))-\operatorname{det}(L(G)\{X\})\right)
\end{aligned}\right.
$$

In these formulae $l_{i i}$ denotes a diagonal term of $L(G)$ and $Q_{k, n}$ denotes the set of all the $C_{n}^{k}$ strictly increasing sequences of size $k(2 \leq k \leq n)$ obtained from the set $\{1,2, \ldots, n\}$. For any $n \times n$ matrix $M$ and for $X \in Q_{k, n}$ let $M[X]$ be the $k \times k$ principal sub-matrix of $M$ corresponding to $X . M\{X\}$ is the $n \times n$ matrix:

$$
M\{X\}=\left(\begin{array}{cc}
M[X] & 0_{k} \\
0_{k} & I_{n-k}
\end{array}\right)
$$

where $I_{n-k}$ is the identity matrix of size $n-k$ and $0_{k}$ is the null matrix of size $k . M\{X\}(i)$ is the matrix obtained from $M\{X\}$ by removing the $i$-th row and the $i$-th column.

An important property of the second immanantal polynomial associated with a graph is that it is preserved under similarity permutation [27]:

$$
d_{2}(x I-L(G))=d_{2}\left(x I-P L(G) P^{-1}\right)
$$

Therefore, a necessary condition for two graphs to be isomorphic is that they have the same second immanantal polynomial. However, it is not a sufficient condition. In practice, however, there have been found very few examples of non-isomorphic graphs that have the same second immanantal polynomial [25].

\subsection{An example}

In order to illustrate the above formalism let us consider two simple binary graphs and let us outline the computation of their associated second immanantal polynomials. An example of two 4-node binary graphs are shown on Figure 1. The Laplacian matrices are given by equation (3) and they are easy to compute:

$$
\begin{aligned}
L(G)_{1}= & \left(\begin{array}{rrrr}
3 & -1 & -1 & -1 \\
-1 & 1 & 0 & 0 \\
-1 & 0 & 1 & 0 \\
-1 & 0 & 0 & 1
\end{array}\right) \\
L(G)_{2} & =\left(\begin{array}{rrrr}
2 & 0 & -1 & -1 \\
0 & 1 & -1 & 0 \\
-1 & -1 & 3 & -1 \\
-1 & 0 & -1 & 2
\end{array}\right)
\end{aligned}
$$



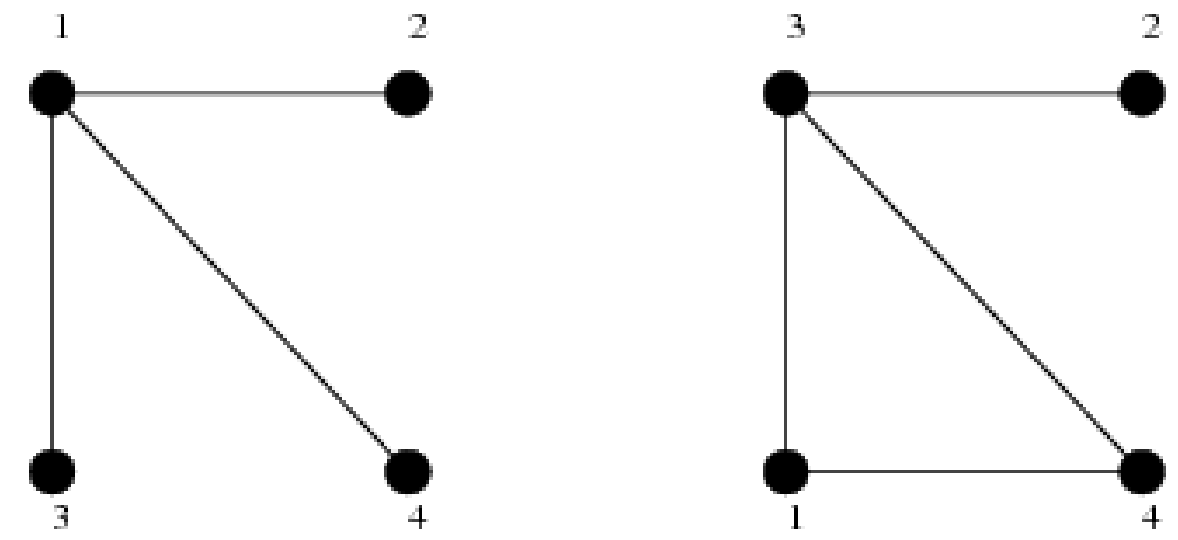

Figure 1: These two graphs differ by one edge but their associated second immanantal polynomials are quite different.

For $n=4$ the sets of all the $C_{4}^{k}$ strictly increasing sequences of size $k(2 \leq k \leq 4)$ are:

$$
\begin{aligned}
Q_{2,4} & =\{(1,2),(1,3),(1,4),(2,3),(2,4),(3,4)\} \\
Q_{3,4} & =\{(1,2,3),(1,2,4),(1,3,4),(2,3,4)\} \\
Q_{4,4} & =\{(1,2,3,4)\}
\end{aligned}
$$

It is straightforward to compute the matrices $L(G)\{X\}$ and the matrices $L(G)\{X\}(i)$, for $X \in Q_{k, 4}$. For example, $L(G)_{1}\{(1,2)\}$ is a $4 \times 4$ matrix obtained by appending the first two rows and first two columns of $L(G)_{1}$ with $I_{2}$ and $0_{2}$ as follows:

$$
L(G)_{1}\{(1,2)\}=\left(\begin{array}{rrrr}
3 & -1 & 0 & 0 \\
-1 & 1 & 0 & 0 \\
0 & 0 & 1 & 0 \\
0 & 0 & 0 & 1
\end{array}\right)
$$

$L(G)_{1}\{(1,2)\}(2)$ is a $3 \times 3$ matrix obtained from $L(G)_{1}\{(1,2)\}$ by removing its 2 nd row and 2 nd column:

$$
L(G)_{1}\{(1,2)\}(2)=\left(\begin{array}{ccc}
3 & 0 & 0 \\
0 & 1 & 0 \\
0 & 0 & 1
\end{array}\right)
$$

After some straightforward computation we obtain the following coefficients for the associated polynomials:

$$
\begin{aligned}
& d_{2}\left(x I-L(G)_{1}\right)=3 x^{4}-18 x^{3}+33 x^{2}-24 x+6 \\
& d_{2}\left(x I-L(G)_{2}\right)=3 x^{4}-24 x^{3}+105 x^{2}-68 x+24
\end{aligned}
$$

One may also compute the characteristic polynomials associated with the Laplacian matrices, i.e.:

$$
\begin{aligned}
\operatorname{det}\left(x I-L(G)_{1}\right) & =x^{4}-6 x^{3}+9 x^{2}-4 x \\
\operatorname{det}\left(x I-L(G)_{2}\right) & =x^{4}-8 x^{3}+19 x^{2}-12 x
\end{aligned}
$$

From this example it is obvious that the second immanantal polynomial is a richer graph description than just the characteristic polynomial. 


\subsection{Weighted graphs}

In general, binary graphs are not sufficient for describing the structure of either images or objects. Weighted graphs are graphs which have a weight $w_{i j}$ associated with the edge linking nodes $i$ and $j$. The definition of the Laplacian matrix may easily be extended to weighted graphs, as follows:

$$
l_{i j}^{w}= \begin{cases}D_{i} & \text { if } i=j \\ -w_{i j} & \text { if there is a weighted edge between node s } i \text { and } j \\ 0 & \text { if there is no edge between nodes } i \text { and } j\end{cases}
$$

with $D_{i}$ being equal to the sum of the weights of the edges meeting at the node $i$ :

$$
D_{i}=\sum_{j=1}^{n} w_{i j}
$$

This matrix has the same properties as the Laplacian matrix associated with a binary graph it is symmetric semidefinite positive and of rank $n-1$ which makes it suitable for computing the $d_{2}$-polynomial.

\section{Graph indexing}

The graph characterization in terms of the coefficients of the second immanantal polynomial allows one to assert whether two graphs with the same number of nodes $(n)$ are "equal". The difference between two graphs $G_{1}$ and $G_{2}$ is given by the formula:

$$
\operatorname{Diff}\left(G_{1}, G_{2}\right)=\sum_{k=1}^{n}\left(c_{k}^{1}-c_{k}^{2}\right)^{2}
$$

where $c_{k}^{i}$ is the $k$-th coefficient of the second immanantal polynomial associated with graph $i$. Since we assume that the above equation is valid only for graphs with the same number of nodes, $c_{0}$ has been skipped out from the summation.

In the case of indexing we are faced with the problem of comparing an image graph with many database graphs and of deciding which are the few graphs in the database that are equal to the image graph. In that case the graph difference mentioned above is not efficient. One way to implement indexing efficiently is to use hashing [30]. Hashing can be briefly described as follows. Each database object has a numerical key associated with it. Then a hash function maps this key onto the address of an array of a manageable size. The address thus computed for an object is also called the hash-code of that object. In practice, hashing is composed of an off-line step (database construction) and a runtime step (indexing):

- Database construction consists of computing a hash-code for each object to be stored in the database. Several objects may well have the same address (hash-code). Therefore a list of objects will be associated with each address. The database takes therefore the form of an array (or a hash-table), a list of objects being stored at each array-element address.

- Indexing consists of computing the address (hash-code) of an unknown object in order to determine whether this object is in the hash-table or not. 
Since a graph may be described by the integer coefficients of a polynomial, these coefficients may well be viewed as the hash-codes of the graph. Hence, a graph with $n$ nodes can be mapped onto $n$ hash tables. For reasons that will be made clear below, the size of the graphs we deal with varies between 5 nodes and 10 nodes. Within this size range the second immanantal polynomial uniquely characterizes binary and weighted graphs. It follows that graph indexing will become an efficient technique because the hashing will have very few collisions associated with it.

Polynomial characterization of graphs combined with graph indexing will eventually allow us to perform object recognition by indexing. However, two important issues need be raised before we describe a practical object recognition system: object representation and database organization.

\subsection{Object representation}

Object representation has been thoroughly studied in Computer Vision and a recent paper by Flynn \& Jain [11] provides a good state of the art. In general there are two possible representation classes: Object frame centered and viewer frame centered representations. Within our approach we use a representation that is not tight to a specific coordinate frame. An object is mainly described by a list of characteristic views. In the representation that we use the definition of a characteristic view $(\mathrm{CV})$ should be understood in a broad sense. It is a network of object features and feature relationships that are simultaneously visible from some viewpoint. Such a representation is by no means limited to the aspect graph representation of an object. The features in the network may well be either 2-D or 3-D, object-centered or viewer-centered. The important characteristic here is not as much the dimensionality of the features or the coordinate frame to which they relate, but instead, the intrinsic properties of the feature network. As we already mentioned, such a feature network can be conveniently represented by a weighted graph.

However, the data associated with some view of an object rarely encodes a whole characteristic view associated with that object. The data are corrupted by noise, occlusions, self occlusions, and accidental alignments. Therefore it will not be very useful to directly store in the database the graph associated with a characteristic view. Instead, each characteristic view is further decomposed in a number of, possibly overlapping, "smaller" views or subviews, where each such subview is in fact associated with a subgraph of the graph describing the characteristic view. There are several reasons in support of the decomposition of a characteristic view into a number of subviews:

- Following the results of section 2, one can compare only graphs with the same number of nodes. Since a graph extracted from the data has rarely the same number of nodes as the graphs associated with the characteristic views of the objects to be recognized, one may still attempt to compare an unknown-object-view with a characteristic-view by comparing subgraphs associated with subviews of theses views.

- The cost of the computation of the coefficients of the second immanantal polynomial is proportional to $n^{4}$, where $n$ is the number of nodes of the graph. Since "raw" characteristic views may have a large number of features associated with them, it may not be efficient to compute polynomial characterizations for very large graphs.

- Consider a data graph that is composed of a large network of features. It is very unlikely that such a large data graph belongs to a unique object. Recognition based on such large graphs will fail because these graphs are not present in the database. 
It is therefore clear that object recognition by indexing must adopt a representation such that each characteristic view is decomposed in a number of subviews or subgraphs. A compromise must be made concerning the size of these subgraphs: Very small subgraphs are too ambiguous because they do not capture information that is object specific while large subgraphs are difficult to extract from the data. At the limit, a single-node graph belongs to all the objects in the database and indexing is useless in this case. At the other extreme, a very large graph encapsulates more than one object and the indexing process will fail to find this graph in the database.

\subsection{Database organization and the indexing mechanism}

Following the above discussion, the organization of the database follows a three-layer structure. A first layer contains a list of graphs of various sizes that are organized in hash tables. The second layer contains characteristic views. A third layer contains descriptions of the object themselves. This structure is shown on Figure 2.

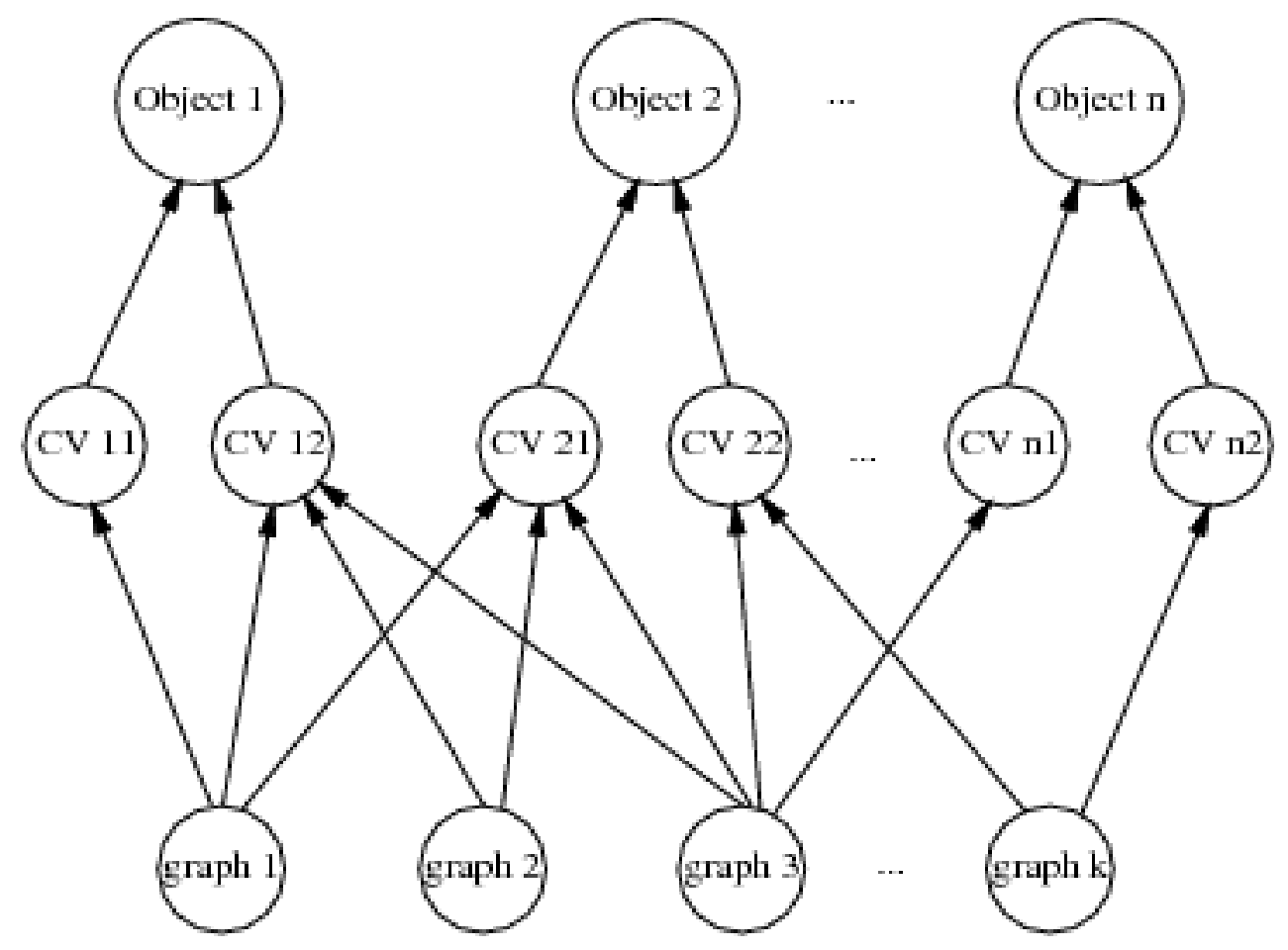

Figure 2: The database has a three layer structure: graphs, characteristic views, and objects. An object may well have more than 2 characteristic views associated with it.

It is clear that a graph belongs to several characteristic views and hence, it may belong to several objects. Therefore, an image graph that matches a graph in the database provides handles to more than one object. The interesting feature of this three-layer organization is that the graph list grows sub-linearly with the number of characteristic views.

The indexing mechanism proceeds as follows. Let's suppose that an unknown object view has to be recognized. First, this unknown view is decomposed into subviews and a graph is associated with each subview. Polynomial characterizations are computed for these unknown graphs. Based on these characterizations and on the hashing technique just described, each unknown graph is 
assigned a unique graph in the database. As a consequence, a list of characteristic views may now be associated with each unknown graph in the image. In other terms, each unknown graph votes for a number of characteristic views. This process is repeated for each unknown graph belonging to the unknown view. The characteristic view that received the largest number of votes is the model that best matches the unknown view.

Consider, for example the database depicted on Figure 2 and suppose that two unknown graphs are assigned graph1 and graph2 respectively. It follows that two characteristic views (CV12 and CV21) received 2 votes while one characteristic view (CV11) received only one vote.

\section{2-D characteristic views of polyhedral objects}

In order to recognize an object with the method described above, one has to represent that object in terms of a few characteristic views and to describe each such characteristic view in terms of a set of weighted graphs. Unlike solutions that consists of computing characteristic views from CAD object descriptions, our approach for obtaining these views is to gather as many images of an object as characteristic views are needed for describing that object unambiguously. Although in this paper we use an ad-hoc technique, more formal methods may be found in [31], [32]: The authors define a set of characteristic views of a polyhedral object by partitioning a large set of object views into small sets of characteristic views.

The task of decomposing a characteristic view into subgraphs is not an easy one. The most general approach would be to implement a graph decomposition method. For example, one may attempt to partition a graph into a pre-specified number of subgraphs such that the number of connections (edges) between the subgraphs is minimized [33]. Here we prefer a more pragmatic solution. For example, one may consider all the nodes of a characteristic view and form subgraphs around each such node. A subgraph is thus formed by this node as well as the nodes that are at a distance less or equal than $p$ edges away from this node. It turns out that this redundant decomposition of a characteristic view in small graphs of various sizes is one key to the success of our recognition method. Indeed, small perturbations in the topology of a view (due essentially to noise or to segmentation errors) will not affect the topology of all the subgraphs extracted from this view.

In the particular case of polyhedral objects, if the degree of a node is, on an average, equal to 3 and for $p \leq 2$ then the number of nodes of the associated subgraphs varies between 5 and 10 . Figure 3 shows a characteristic view of a simple polyhedral object and some subgraphs extracted from this view with $p=2$.

As it has already been discussed, the topology of a characteristic view is not sufficient for describing the view unambiguously. For example, Figure 4 shows six different binary graphs which are isomorphic (they have the same topology). Clearly, one would like to be able to state that the top three graphs are different and the bottom three ones are identical. In other words, the top three ones do not look the same, although they have the same topology. The question of how to describe the 2-D appearance of a polyhedral object has already been addressed (see for example [34]) but the question of how to represent such an appearance with a weighted graph has not.

One way to label an edge is to characterize it according to the structure of the vertices at each endpoint of that edge. If we consider polyhedra that have at most 3 edges meeting at a vertex, then we obtain a catalogue of possible edge structures or edge appearances. It is sufficient to assign 

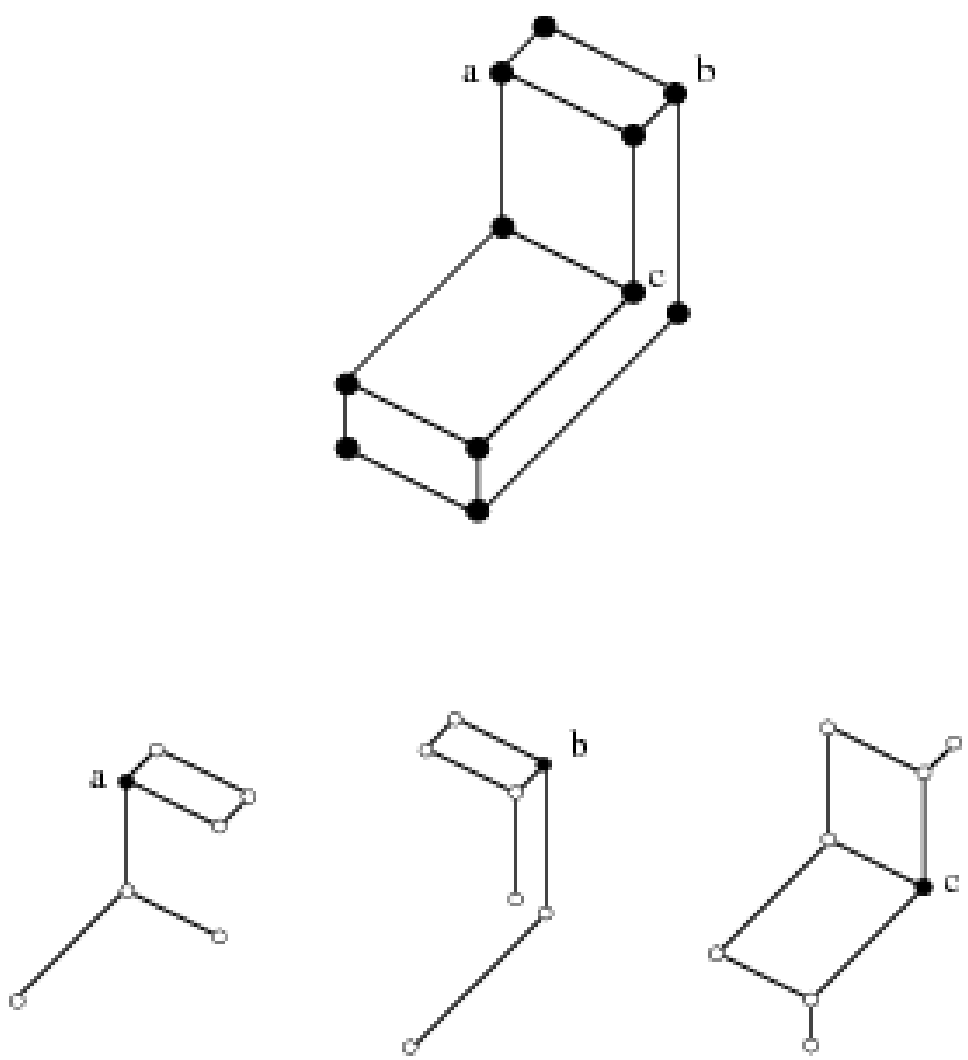

Figure 3: An example of a characteristic view of an object and a few graphs extracted from this view.

labels to these various appearances and to associate a weight to each label. Figure 5 shows an exhaustive catalogue of edge appearances and their weights.

There are three possible vertex structures: a 2-edge vertex or an "L", and two 3-edge vertices, an "Y" and an "Arrow". Since an edge divides the plane into two regions - the left side and the right side - we obtain the following list of features that allows the labelling and the weighting of an edge (see Figure 6 for an example of an edge labelled "15"):

- the type of the first vertex (Arrow);

- the type of the second vertex (Arrow);

- the number of edges associated with the first vertex lying on the left side of the edge; (1)

- the number of edges associated with the first vertex lying on the right side of the edge (1);

- the number of edges associated with the second vertex lying on the left side of the edge (2);

- the number of edges associated with the second vertex lying on the right side of the edge (0). 

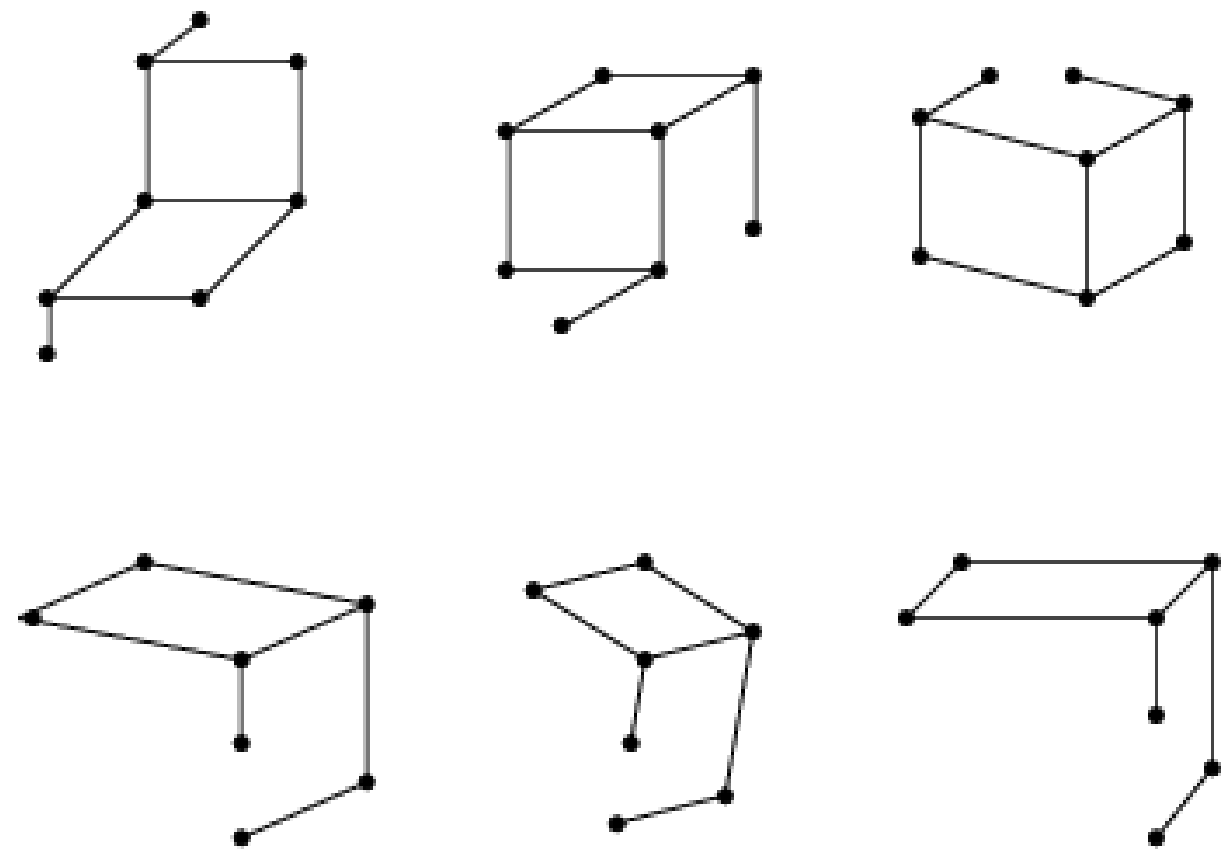

Figure 4: Top - three graphs having the same topology. Bottom - three other graphs having the same topology and the same appearance.

\section{$5 \quad$ Image processing}

In this section we describe the process by which a number of graphs is extracted from an image. This graph extraction process has some similarities with feature grouping since its goal is to provide a few "key" image features and reduce the complexity of the object recognition process. Image processing starts with extracting edges and with approximating these edges with straight lines. Junctions are next extracted. The junctions and the straight lines form a network of features, or a graph - the image graph.

If the scene is composed of just one object, then this image graph corresponds, up to some noise, to a view of that object. Single object scenes are used for building the database of characteristic views and it has been previously described.

If the scene is composed of more than one object and if the background is not uniform, then the image graph has to be further processed in order to be split into smaller graphs. Each small graph thus obtained is examined in order to decide whether it should be considered for recognition or not. To summarize, the process of extracting graphs from an image comprises the following steps:

1. image graph extraction;

2. image graph splitting, and

3. graph evaluation.

Step 1. Image graph extraction has been briefly outlined at the beginning of this section and is described in detail in [35].

Step 2. Image graph splitting is based on a number of heuristics: 

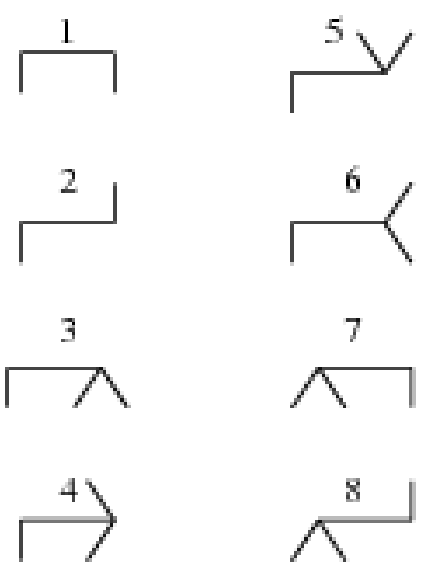
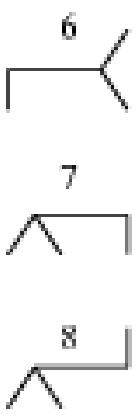
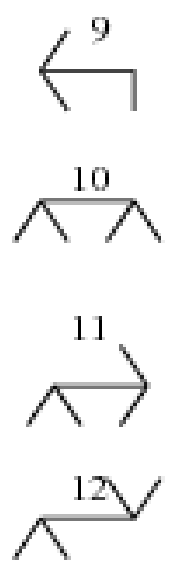
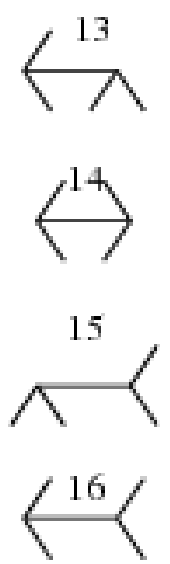
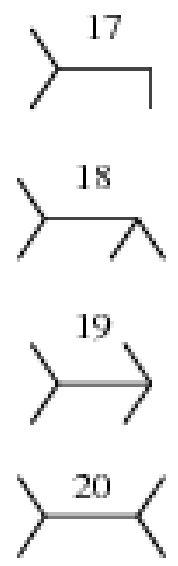

Figure 5: An exhaustive catalogue of the possible appearances of the edges of a polyhedral object that has, at most, 3 edges meeting at a vertex.

2.1 Isolated and "dangling" edges are thrown out.

2.2 It is assumed that "T" junctions arise from occlusions (an object in front of another object, an object in front of some background, or a self occlusion). Hence, the image graph is cut off at $\mathrm{T}$ junctions. Notice that this process may produce isolated edges which are immediately thrown out.

2.3 Sequences of collinear edges are assumed to arise from the same physical edge and hence, collinear edges are fused into a unique edge.

2.4 Finally, the image graph is decomposed into connected components.

Step 3. Graph evaluation considers each connected component, one by one, and evaluates it in order to decide whether it should be further considered for recognition or thrown out. Let $n$ be the number of nodes of a graph and let $d_{i}$ be the degree of node $i$, i.e., equation (3). The quantity:

$$
f(G)=\frac{1}{n} \sum_{i=1}^{n} d_{i}
$$

allows one to measure the complexity of a graph. It is straightforward to notice that for $f(G)=2$ the graph has at most one cycle. Since graphs without cycles are not really relevant, one may consider only graphs for which:

$$
f(G) \geq 2
$$

The graphs that don't satisfy this constraint are consider irrelevant and therefore they are thrown out.

Let us illustrate with an example the graph extraction process that we just described. Figure 7 shows an intensity image (top-left) and a network of lines and junctions extracted from this image (top-right) from which isolated and dangling lines are removed (middle-left). The next image (middle-right) shows the T-junctions that are removed from the list of junctions. This T-junction removal process produces isolated lines and dangling lines on one hand (which are removed) and 


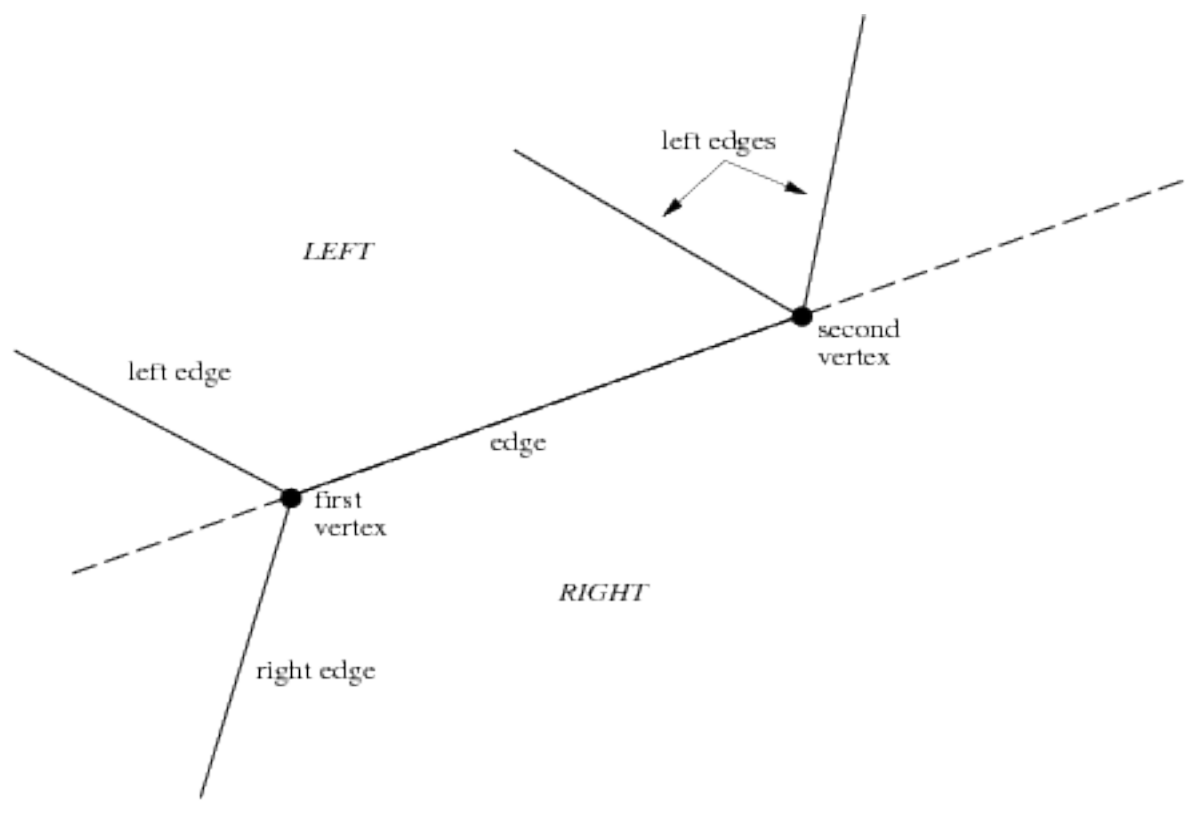

Figure 6: The labelling of an edge depends on the structure of the two vertices at each endpoint of that edge (see text).

collinear lines which are fused into a unique line on the other hand (bottom-left). We are left now with a number of connected image graphs. Each such connected component is evaluated according to the Step 3 just above. The latter process leaves 4 connected components in the image (bottomright). These remaining image graphs will be further decomposed into subgraphs in order to be used by the indexing process. The decomposition of the 4 image graphs into subgraphs is not shown.

\section{Experiments}

All the object recognition experiments that we performed used the same database, namely 20 characteristic views associated with 6 objects, as shown on Figures 8 and 9 . All the objects in this database are 3-D polyhedral shapes with one exception. The database was built by showing each object, view by view, to the camera and by applying the graph extraction process described in section 5 .

We carried out many experiments in which the input image varied from a very simple one with just one object against an uniform background to more complex images with many objects against a non-uniform background.

In one such experiment we grabbed 9 images of the same scene and we processed these images identically (with exactly the same segmentation parameters). Figure 10 shows these 9 images where the camera position and orientation varies with respect to the observed scene. Figure 11 shows the graphs extracted from these images. The images are numbered 1 to 9 from left to right and top to bottom.

Table 1 summarizes the results of recognizing the two objects based on the two graphs (labelled "0" and "1") extracted from the 9 images. The figures in this table correspond to the scores (number 


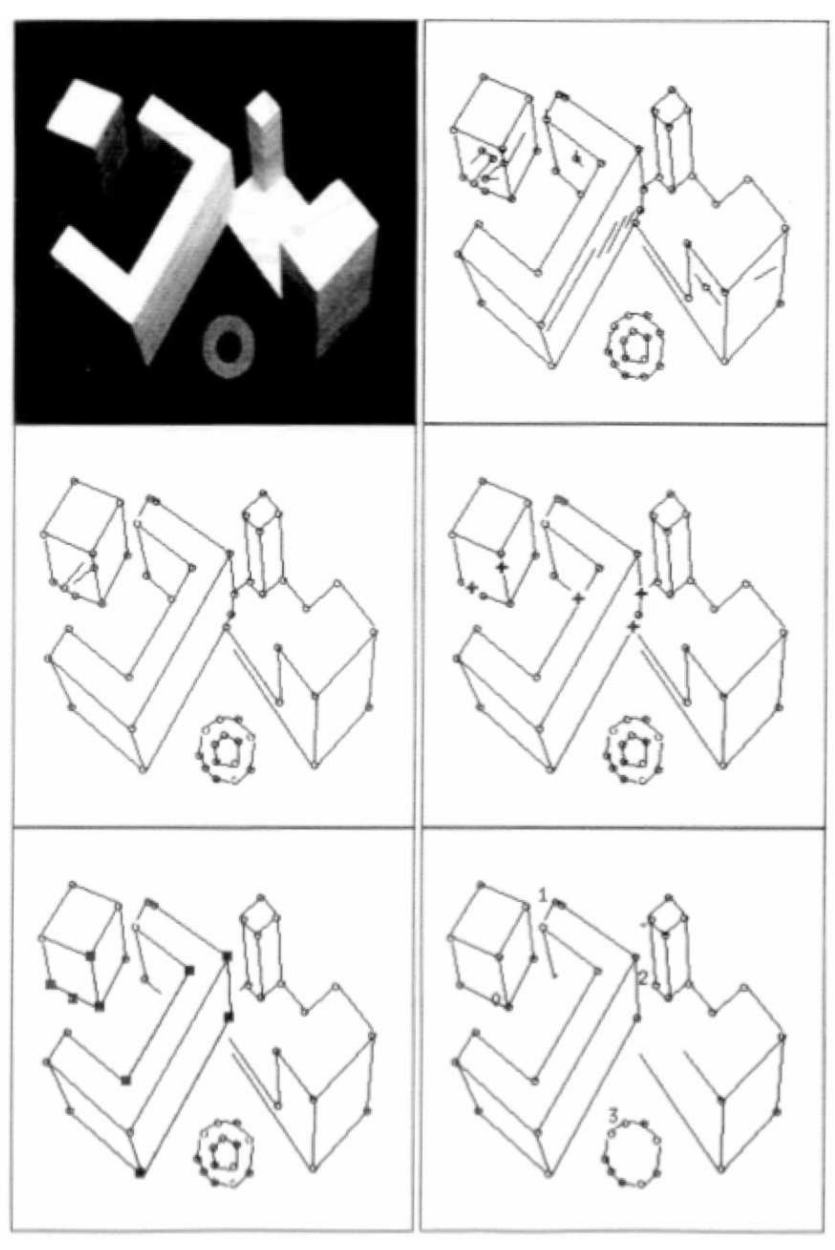

Figure 7: An example of extracting a set of 4 image graphs from an intensity image (top-left): Lines and junctions are detected (top-right), isolated and dangling lines are removed (middle-left), T-junctions are detected and removed (middle-right), collinear lines are fused into longer lines (bottom-left), the remaining graphs are evaluated and four of them survive (bottom-right).

of votes) received by each characteristic view when the image graphs are indexing the database. For each image graph the table records its highest score, sometimes the two highest scores. The first object (the graph labelled "0") has been correctly recognized 7 times and incorrectly recognized twice (images 5 and 9). Notice the high scores (between 16 and 24) obtained in the case of a correct recognition in comparison with the less high scores (between 6 and 8) obtained in the case of an incorrect recognition.

The same phenomena can be observed with the second object (the image graph labelled "1") which has been correctly recognized 6 times (the score varies between 8 and 25) and incorrectly recognized 3 times (the score varies between 4 and 7). An interesting remark is that all 5 incorrect recognitions assigned the same characteristic view to the unknown image graphs, namely the view labelled "pieceLd02m" (the bottom-rightmost view on Figure 9). One may notice the poor segmentation associated with this characteristic view.

The recognition results reported above are barely affected if one increased the size of the database of characteristic views by adding views of very different objects. Of course, if the database 
contains two very similar objects, the system will fail to discriminate between these two objects.
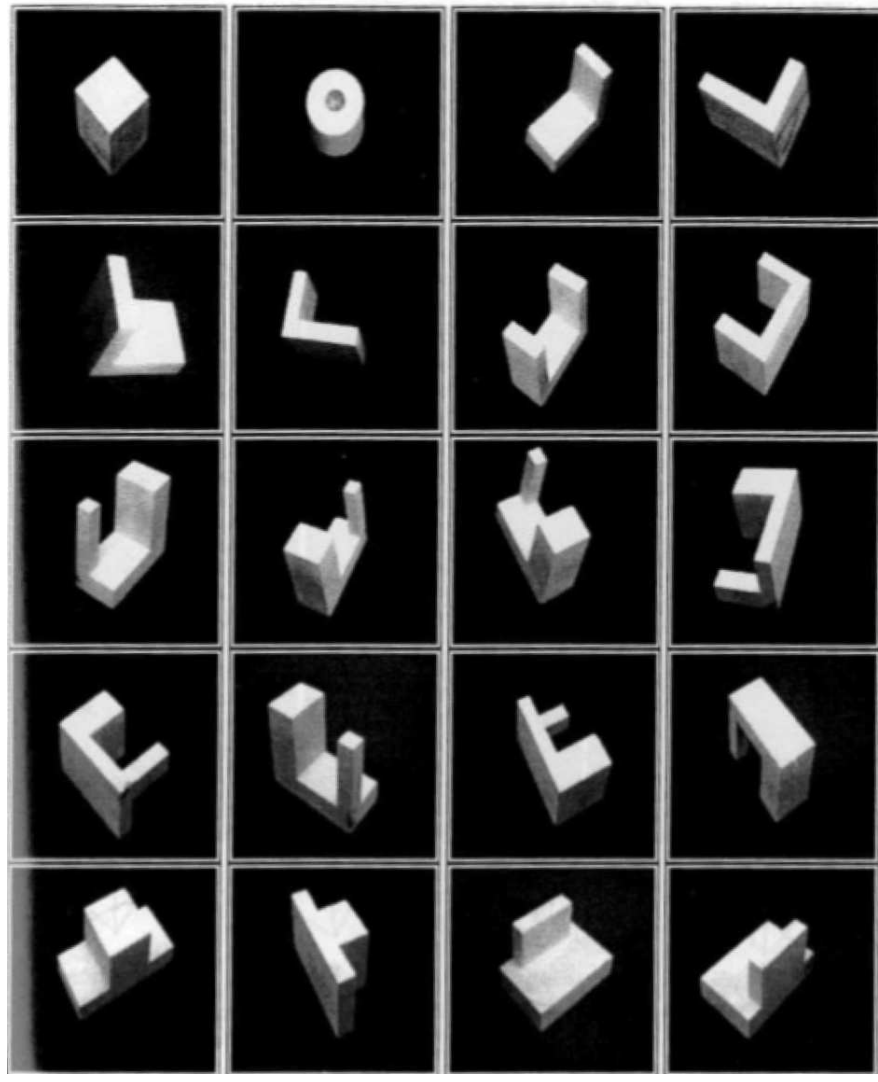

Figure 8: This figure shows the intensity images of 20 characteristic views associated with 6 objects. These views and objects constitute the database.

\section{Discussion}

Unlike the prevailing paradigm in computer vision that suggests image-feature-to-object-feature matching to solve for object recognition, we described an approach that uses an indexing technique to compare objects in an image with objects in a database. Our method doesn't rely neither on precise knowledge about the geometry of the objects nor on reliable feature-to-feature assignments. Instead we describe both the images and the models by weighted graphs and we compare these graphs through their polynomial characterization, namely the second immanantal polynomial of the Laplacian matrix of a graph. This graph comparison was implemented in two steps (off-line and on-line): a database construction step (model graphs are stored in hash tables) and an indexing step (an image graph indexes in the pre-stored hash tables).

It is worthwhile to notice that, in the past, polynomial characterization of graphs has been used to represent and identify the topology of molecules [36]. At our knowledge, the graph theory literature doesn't describe any attempt to generalize polynomial characterizations to weighted graphs. It turns out that, at least for our purposes, this generalization is straightforward since the Laplacian matrix of a weighted graph has the same mathematical properties as the Laplacian matrix of a binary graph. 


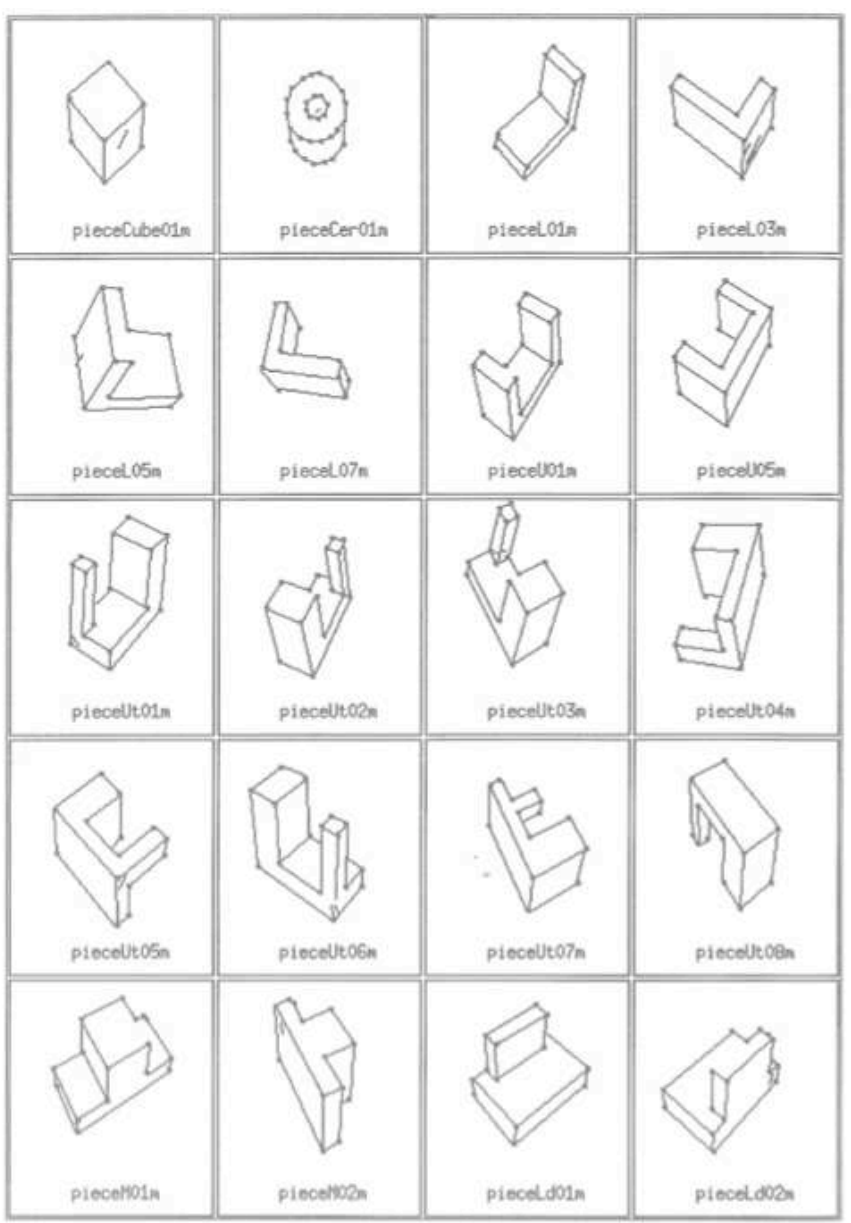

Figure 9: The 20 graphs (lines and junctions) extracted from the previous images. These 20 graphs are decomposed into subgraphs (the decomposition is not shown) and stored in the hash-tables associated with the database.

We believe that our indexing scheme based on this algebraic graph representation has a promising potential in computer vision and may provide in the future an interesting paradigm for indexing.

\section{References}

[1] A.P. Ambler, H.G. Barrow, C.M. Brown, R.M. Burstall, and R.J. Popplestone. A versatile computer-controlled assembly system. In Proc. Third International Joint Conference on Artificial Intelligence, pages 298-307, Stanford University, CA, USA, August 1973.

[2] D. H. Ballard and C. M. Brown. Computer Vision. Prentice Hall Inc., Englewood Cliffs, New Jersey, 1982.

[3] R. C. Bolles and R. A. Cain. Recognizing and locating partially visible objects, the LocalFeature-Focus method. International Journal of Robotics Research, 1(3):57-82, 1982. 

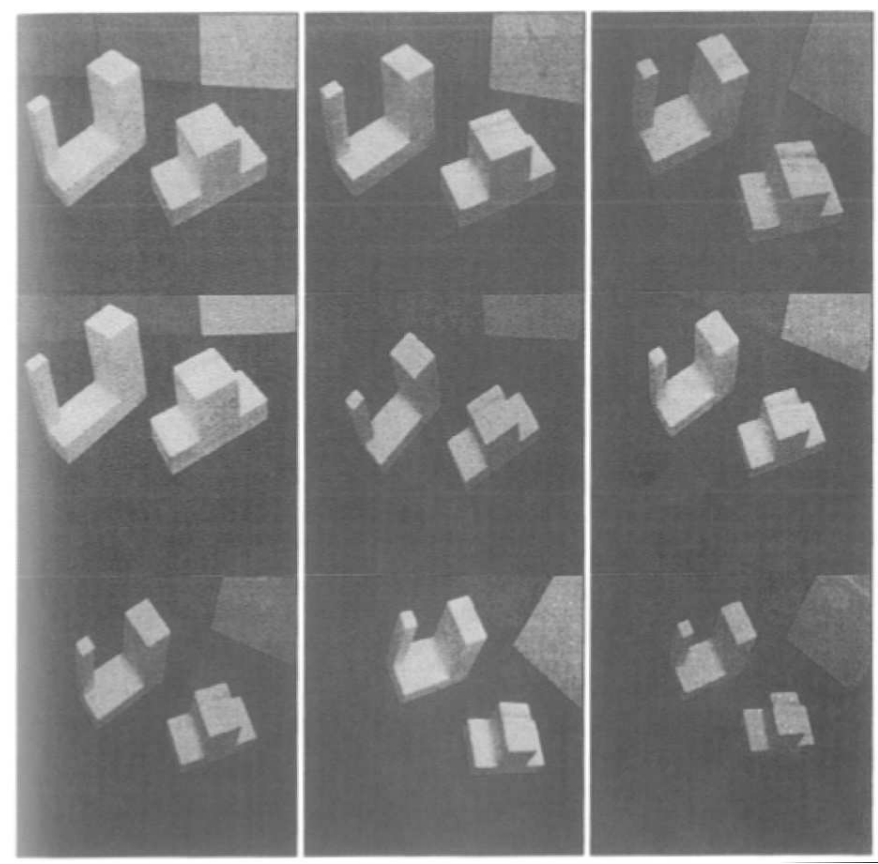

Figure 10: Six images of two objects to be recognized where the camera varies in position and orientation with respect to the two objects.

[4] R. C. Bolles and R. Horaud. 3DPO: A three-dimensional part orientation system. International Journal of Robotics Research, 5(3):3-26, Fall 1986.

[5] O.D. Faugeras and M. Hebert. The representation, recognition, and locating of 3-d objects. International Journal of Robotics Research, 5(3):27-52, Fall 1986.

[6] N. Ayache and O. D. Faugeras. HYPER: A new approach for the recognition and positioning of two-dimensional objects. IEEE Trans. on Pattern Analysis and Machine Intelligence, PAMI8(1):44-54, January 1986.

[7] W.E.L. Grimson and T. Lozano-Perez. Localizing overlapping parts by searching the interpretation tree. IEEE Transactions on Pattern Analysis and Machine Intelligence, PAMI-9(4):469482, July 1987.

[8] C. Goad. Fast 3D model-based vision. In Alex B. Pentland, editor, From Pixels to Predicates, chapter 16, pages 371-391. Ablex Publishing Corporation, Norwood, New Jersey, 1986.

[9] P. J. Flynn and A. K. Jain. BONSAI: 3-D object recognition using constrained search. IEEE Transactions on Pattern Analysis and Machine Intelligence, 13(10):1066-1075, October 1991.

[10] W. Y. Kim and A. C. Kak. 3-D object recognition using bipartite matching embedded in discrete relaxation. IEEE Transactions on Pattern Analysis and Machine Intelligence, 13(3):224251, March 1991.

[11] P. J. Flynn and A. K. Jain. CAD-based computer vision: From cad models to relational graphs. IEEE Transactions on Pattern Analysis and Machine Intelligence, 13(2):114-132, February 1991. 


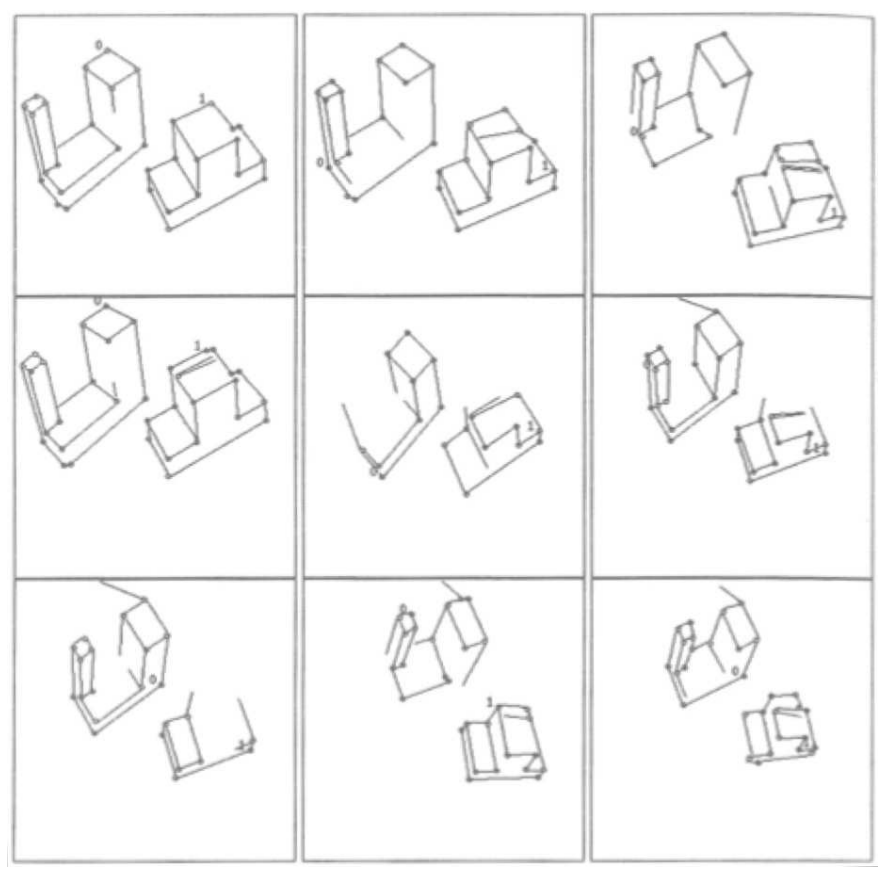

Figure 11: The graphs extracted from the previous images. Notice that the noise corruption of these graphs varies a lot even if there is only a small change in camera position and orientation.

[12] S. Dickinson, A. Pentland, and A. Rosenfeld. 3-D shape recovery using distributed aspect matching. IEEE Transactions on Pattern Analysis and Machine Intelligence, 14(2):174-198, 1992.

[13] R. Bergevin and M. D. Levine. Generic object recognition: Building and matching coarse descriptions from line drawings. IEEE Transactions on Pattern Analysis and Machine Intelligence, 15(1):19-36, January 1993.

[14] S. Umeyama. An eigendecomposition approach to weighted graph matching problems. IEEE Transactions on Pattern Analysis and Machine Intelligence, 10(5):695-703, May 1988.

[15] M. Hanajik, F. J. Kylstra, and R. G. van Vliet. An analytical approach to the matching of attributed graphs. In Proceedings of the 8th Scandinavian Conference on Image Analysis, pages 419-425, Tromso, August 1993.

[16] H. A. Almohamad and S. O. Duffuaa. A linear programming approach for the weighted graph matching problem. IEEE Transactions on Pattern Analysis and Machine Intelligence, 15(5):522-525, May 1993.

[17] L. Hérault, R. Horaud, F. Veillon, and J-J. Niez. Symbolic Image Matching by Simulated Annealing. In Proceedings British Machine Vision Conference, pages 319-324, Oxford, Great Britain, September 1990.

[18] V. Tresp and G. Gindi. Invariant object recognition by inexact subgraph matching with applications in industrial part recognition. In Proc. International Neural Network Conference, pages 95-98, Paris, July 1990. 


\begin{tabular}{|c|c|c|c|c|c|c|c|}
\hline \multirow{2}{*}{$\begin{array}{l}\text { image } \\
\text { number }\end{array}$} & \multirow{2}{*}{$\begin{array}{c}\text { graph } \\
\text { number }\end{array}$} & \multicolumn{6}{|c|}{ characteristic view } \\
\hline & & Ut01m & Ut02m & M01m & $\mathrm{M} 02 \mathrm{~m}$ & L05m & $\mathrm{Ld} 02 \mathrm{~m}$ \\
\hline \multirow[t]{2}{*}{1} & 0 & $18^{\star}$ & $24^{\star}$ & & & & \\
\hline & 1 & & & $23^{\star}$ & & & \\
\hline \multirow[t]{2}{*}{2} & 0 & $16^{\star}$ & $16^{\star}$ & & & & \\
\hline & 1 & & & $17^{\star}$ & & & \\
\hline \multirow[t]{2}{*}{3} & 0 & & $18^{\star}$ & & & & \\
\hline & 1 & & & $8^{\star}$ & & & \\
\hline \multirow[t]{2}{*}{4} & 0 & $17^{\star}$ & $24^{\star}$ & & & & \\
\hline & 1 & & & $13^{\star}$ & & 10 & \\
\hline \multirow[t]{2}{*}{5} & 0 & & & & 6 & & 7 \\
\hline & 1 & & & $9^{\star}$ & & & \\
\hline \multirow[t]{2}{*}{6} & 0 & $17^{\star}$ & $23^{\star}$ & & & & \\
\hline & 1 & & & 6 & & & 7 \\
\hline \multirow[t]{2}{*}{7} & 0 & & $23^{\star}$ & & & & \\
\hline & 1 & & & & & & 4 \\
\hline \multirow[t]{2}{*}{8} & 0 & & $16^{\star}$ & & & & \\
\hline & 1 & & & 4 & & & 5 \\
\hline \multirow[t]{2}{*}{9} & 0 & & & & 6 & & 8 \\
\hline & 1 & & & $13^{\star}$ & & & \\
\hline
\end{tabular}

Table 1: This table shows the results of recognition for 9 images of the same scene. The figures correspond to scores (number of votes) as a result of the graph indexing process. The scores over-scripted by $a \star$ correspond to a correct recognition.

[19] R. Nevatia and T. Binford. Description and recognition of complex-curved objects. Artifitial Intelligence, 8:77-98, 1977.

[20] G. J. Ettinger. Large Hierarchical Object Recognition Using Libraries of Parmeterized Model Sub-Parts. In Proc. Computer Vision and Pattern Recognition, pages 32-41, Ann Arbor, Michigan, USA, June 5-9 1988.

[21] A. Kalvin, E. Schomberg, J. T. Schwartz, and M Sharir. Two-dimensional model-based, boundary matchig using footprints. The International Journal of Robotics Research, 5(4):38$54,1986$.

[22] Y. Lamdan and H. Wolfson. Geometric hashing: A general and efficient model-based recognition scheme. In Second International Conference on Computer Vision, pages 238-249, Tampa, Florida, USA, December 1988.

[23] F Stein and G. Medioni. Structural hashing: Efficient 3-d object recognition. IEEE Transactions on Pattern Analysis and Machine Intelligence, 14(2):125-145, February 1992.

[24] T. M. Breuel. Adaptive model base indexing. In Proc. DARPA IU Workshop, pages 805-814, 1989. 
[25] D. M. Cvetkovic, M. Doob, and H. Sachs. Spectra of Graphs. Academic Press, New York, 1980.

[26] J. Turner. Generalized matrix functions and the graph isomorphism problem. SIAM J. Appl. Math, 16(3):520-526, May 1968.

[27] R. Merris, K. R. Rebman, and W. Watkings. Permanental polynomials of graphs. Linear Algebra Applications, 38:273-288, 1981.

[28] R. Merris. The second immanantal polynomial and the centroid of a graph. SIAM Journal Alg. Disc. Meth., 7:484-503, 1986.

[29] G. M. Constantine. Graph complexity and the laplacian matrix in blocked experiments. Linear and Multilinear Algebra, 28:49-56, 1990.

[30] R. Sedgewick. Algorithms. Addison-Wesley Publishing Company, Inc., Massachusetts, 1988.

[31] P. Gros and R. Mohr. Automatic object modelization in computer vision. In H. Bunke, editor, Proceedings of the workshop "Advances in Structural and Syntactic Pattern Recognition", Bern, Switzerland, volume 5 of Series on Machine Perception and Artificial Intelligence, pages 385400. World Scientific, August 1992.

[32] P. Gros. Matching and clustering: two steps towards automatic model generation in computer vision. In Proceedings of the AAAI Fall Symposium Series: Machine Learning in Computer Vision: What, Why, and How?, Raleigh, North Carolina, USA, pages 40-44, October 1993.

[33] L. Hérault and J.J. Niez. Neural Networks and Graph K-Partitioning. Complex Systems, 3(6):531-576, December 1989.

[34] T. Kanade. Recovery of the 3D shape of an object from a single view. Artificial Intelligence, 17(1-3):409-460, August 1981.

[35] R. Horaud, F. Veillon, and Th. Skordas. Finding geometric and relational structures in an image. In O. Faugeras, editor, Computer Vision - ECCV 90, Proceedings First European Conference on Computer Vision, Antibes, France, pages 374-384. Springer Verlag, April 1990.

[36] Y. Kudo, T. Yamasaki, and S. Sasaki. The characteristic polynomial uniquely represents the topology of a molecule. Journal of Chemical Documentation, 13(4):225-227, 1973. 